\title{
Investigation of hypoglycemic, hypolipidemic and anti-nephritic activities of Paecilomyces tenuipesN45 in diet/streptozotocin-induced diabetic rats
}

\author{
CHUNGANG LIU ${ }^{1}$, XUERUI ZENG ${ }^{1}$, YI LI ${ }^{1}$, HONGLI MA ${ }^{1}$, JINGJING SONG ${ }^{1}$, \\ YUHUAN LI ${ }^{1}$, YULIN ZHOU ${ }^{1}$, ROBERT J. LEE ${ }^{1,2}$ and DI WANG ${ }^{1,3}$ \\ ${ }^{1}$ School of Life Sciences, Jilin University, Changchun, Jilin 130012, P.R. China; ${ }^{2}$ Division of Pharmaceutics, \\ College of Pharmacy, The Ohio State University, Columbus, OH 43210, USA; ${ }^{3}$ Zhuhai College of Jilin University, \\ Jilin University, Zhuhai, Guangdong 519041, P.R. China
}

Received December 25, 2015; Accepted February 2, 2017

DOI: $10.3892 / \mathrm{mmr} .2017 .6311$

\begin{abstract}
Due to its pharmacological activities, Paecilomyces tenuipes has previously been used as a folk medicine in Asia. The primary aim of the present study was to investigate the hypoglycemic, hypolipidemic and anti-nephritic effects of $P$. tenuipes N45 aqueous extracts (PTNE) in a high fat diet/streptozotocin-induced diabetic rat model. The rats were treated with $120 \mathrm{mg} / \mathrm{kg}$ of metformin or $0.04,0.2$ or $1.0 \mathrm{~g} / \mathrm{kg}$ PTNE for 4 weeks. The hypoglycemic activity of PTNE was confirmed by the observation of reduced fasting blood glucose level and by partially normalized oral glucose tolerance. PTNE reduced total cholesterol and triglyceride content, and balanced the levels of low-density and high-density lipoproteins. The suppressive effects of PTNE on creatinine, blood urea nitrogen, interleukin (IL)-2, IL-6 and nuclear factor- $\kappa$ B levels indicated its ability to provide protection against diabetic nephropathy. PTNE treatment increased superoxide dismutase, malondialdehyde and glutathione peroxidase levels, suggesting that its anti-diabetic and anti-nephropathic activities may be associated with the prevention of oxidative damage during type 2 diabetic mellitus. The findings of the present study provided experimental evidence for the application of Paecilomyces tenuipes N45 on the treatment of type 2 diabetic mellitus.
\end{abstract}

Correspondence to: Dr Di Wang, School of Life Sciences, Jilin University, 2699 Qianjin Street, Changchun, Jilin 130012, P.R. China E-mail: jluwangdi@outlook.com

Professor Robert J. Lee, Division of Pharmaceutics, College of Pharmacy, The Ohio State University, 281 West Lane Avenue, Columbus, OH 43210, USA

E-mail: lee.1339@osu.edu

Key words: Paecilomyces tenuipes, diabetes mellitus, hypoglycemic, hypolipidemic, anti-oxidant, anti-nephropathy

\section{Introduction}

Diabetes mellitus, as a chronic endocrine metabolic disease with a high incidence of complications, is a major health risk worldwide (1). As a chronic disease, micro- and macro-vascular complications frequently occur in patients suffering from diabetes mellitus. In addition, complex metabolic disorders involving lipids, carbohydrates and proteins are frequently observed (2). Patients with type 2 diabetes mellitus present with a more aggressive course of disease, and an increased risk for early hypertension and nephropathy compared with patients with type 1 diabetes mellitus. A combination of increased blood glucose levels and organ damage caused by insulin secretion deficiency can result in various pathological complications, including nephropathy (3). Diabetic nephropathy has been demonstrated to be caused by chronic inflammation (4). Activation of nuclear factor $-\kappa \mathrm{B}(\mathrm{NF}-\kappa \mathrm{B})$ was demonstrated to be involved in the pathogenesis of diabetic nephropathy (5).

Standard therapies for diabetes focus on blood glucose regulation and often fail to control the associated complications (6). Certain widely used medications for diabetic management have demonstrated undesirable adverse effects, making further searching for safer and more efficacious treatments of diabetes mellitus urgently required.

Herbal medicines have demonstrated anti-diabetic activities and the ability to reduce diabetic complications (7). For example, Cordyceps militaris polysaccharide-enriched fraction was revealed to have a hypoglycemic effect on diabetic rats (8). Paecilomyces tenuipes, a well-known Chinese medicinal entomopathogenic fungus, has a long history of medicinal use in Asia (9). The anti-depressant, anti-tumor and immunomodulatory activities of $P$. tenuipes have been confirmed in previous studies $(10,11)$. Furthermore, $P$. tenuipes has been demonstrated to improve lipid profiles and lipid peroxidation (12). An improved mutant strain named $P$. tenuipes $\mathrm{N} 45$, with an elevated growth rate and polysaccharide content, was obtained via chemical mutagenesis in the present study. The strain has been successfully preserved in the China Center for Type Culture Collection (CCTCC; no. M2011145). P. tenuipes N45 has previously been 
demonstrated to have anti-hyperglycemic, anti-hyperlipidemic and anti-oxidant effects in alloxan-induced diabetic rats (13). Diabetes mellitus caused by alloxan, which destroys the $\beta$ cells of the pancreas, is pathologically similar to type 1 diabetes mellitus $(14,15)$. It was therefore hypothesized that $P$. tenuipes N45 may display similar beneficial effects on type 2 diabetes mellitus.

The present study aimed to investigate the hypoglycemic, hypolipidemic and anti-inflammatory activities of $P$. tenuipes N45 aqueous extract (PTNE) in a high-fat diet and streptozotocin (STZ)-induced rat model. Following 4 weeks of treatment with PTNE, several indexes associated with oxidation resistance, hypoglycemic, hypolipidemic and anti-nephropathy activities were measured. These data supported the potential use of PTNE as an adjuvant therapy for type 2 diabetes mellitus.

\section{Materials and methods}

Submerged fermentation of P. tenuipes N45. Spores collected from cultured P. tenuipes Pt 196 (RCEF 4339; Anhui Agricultural University, Anhui, China) were washed twice with phosphate buffered saline (PBS) and adjusted to $1 \times 10^{7} / \mathrm{ml}$. The spores were incubated in $1 \mathrm{mg} / \mathrm{ml}$ nitrosoguanidine PBS solution at $25^{\circ} \mathrm{C}$ for $12 \mathrm{~min}$ and then cultured in solid potato dextrose agar medium 96-well plates. For the selection of mutants, individual strains were cultured in $250 \mathrm{ml}$ flasks. By comparing the dry mycelium weight and contents of effective components (adenosine and polysaccharide) of three consecutive cultivated generations, $P$. tenuipes N45 (CCTCC no. M2011145) was obtained as the optimal strain. $P$. tenuipes N45 was cultured in a rotary shaker incubator $(10 \mathrm{~L}$, Biostat $\mathrm{B}$; Germany) at $150 \mathrm{rpm}$ for 5 days, and the temperature was maintained at $26^{\circ} \mathrm{C}$. The culture medium contained following ingredients: $40 \mathrm{~g} / 1$ glucose, $10 \mathrm{~g} / 1$ peptone and $10 \mathrm{~g} / 1$ yeast extract powder. Mycelium pellets were harvested and lyophilized for further usage.

Crude extract preparation. PTNE was prepared as follows: $100 \mathrm{~g}$ mycelial powder was extracted twice in $5 \mathrm{~L}$ double distilled water at $80^{\circ} \mathrm{C}$ for $3 \mathrm{~h}$. Following centrifugation at $3,500 \times \mathrm{g}$ for $10 \mathrm{~min}$, the supernatant was evaporated under reduced pressure at $55^{\circ} \mathrm{C}$ in a rotary evaporator. The extract was subsequently freeze-dried for further experiments.

Diet/STZ-induced diabetic rat model and drug administration procedure. The following experimental protocol was approved by the Institution Animal Ethics Committee of Jilin University (Changchun, China). A total of 60 Sprague-Dawley male rats (SCXK (JI)-2011-0003) (purchased from Norman Bethune University of Medical Science Jilin University, Jilin, China) were maintained on a 12-h light/dark cycle (lights on $07: 00-19: 00)$ at $23 \pm 1^{\circ} \mathrm{C}$ with water and food available ad libitum.

Rats were fed with standard laboratory diet for 2 weeks. Subsequently, 50 rats weighing 260-300 g were selected for the present study. To induce diabetes, 50 rats were fed with a high fat diet $(68.8 \%$ standard chow, $20 \%$ sucrose, $10 \%$ lard, $0.2 \%$ cholesterol and $1 \%$ salt mixture, purchased from the Lab Animal Center of Jilin University, Jilin, China) for
8 weeks, followed with intraperitoneal injection of $30 \mathrm{mg} / \mathrm{kg}$ STZ once per day for 3 days (16). Rats injected with citrate buffer only were used as the control $(n=10)$. During the experiment, rats were fed with $5 \%$ glucose solution for $4 \mathrm{~h}$ following STZ injection to prevent hypoglycemia. Rats with fasting serum glucose levels between 11 and $26 \mathrm{mmol} / 1$ were placed in the diabetic groups. The diet/STZ-induced diabetic rats were randomly separated into five groups and injected with $10 \mathrm{ml} / \mathrm{kg}$ physiological saline (model group; MD; $\mathrm{n}=10$ ), $120 \mathrm{mg} / \mathrm{kg}$ metformin (Met; positive control group; $\mathrm{n}=10$; met purchased from Beijing Jingfeng Zhiyao Co., Ltd., Beijing, China) and $0.04,0.2$ or $1.0 \mathrm{~g} / \mathrm{kg}$ PTNE (n=10/group) for 4 continuous weeks. Bodyweight was recorded for the duration of the experiment, and fasting blood glucose was monitored using a Glucose Assay kit (Nanjing KeyGen Biotech Co. Ltd., Nanjing, China) on day 28 after PTNE treatment, following $18 \mathrm{~h}$ food deprivation.

Oral glucose tolerance test (OGTT). Following 4 weeks of treatment, an OGTT was performed on the diabetic rats. Following $12 \mathrm{~h}$ fasting, rats were orally treated with physiological saline, $120 \mathrm{mg} / \mathrm{kg}$ Met or $0.04,0.2$ or $1.0 \mathrm{~g} / \mathrm{kg}$ PTNE. After $30 \mathrm{~min}, 2.0 \mathrm{~g} / \mathrm{kg}$ glucose was administered orally to each rat. Blood samples were collected at 0, 0.5, 1 and $2 \mathrm{~h}$, and plasma glucose concentrations were measured. The area under the blood glucose curve (AUC) was calculated according to the following equation (13): AUC = (basal glycemia + glycemia $0.5 \mathrm{~h}) \times 0.25+($ glycemia $0.5 \mathrm{~h}+$ glycemia $1 \mathrm{~h}) \times 0.25+($ glycemia $1 \mathrm{~h}+$ glycemia $2 \mathrm{~h}) \times 0.5$.

Biochemical index analysis. Prior to sacrifice, blood was sampled from the hearts of all the rats, which were placed under anesthesia. Superoxide dismutase (SOD; cat. no. A001-3), glutathione peroxidase (GSH-Px; cat. no. A005), malondialdehyde (MDA; cat. no. A003-4), low-density lipoprotein cholesterol (LDL-C; cat. no. A113-1), high density lipoprotein cholesterol (HDL-C; cat. no. A113-2), total cholesterol (TC; cat. no. A111-1), triglycerides (TG; cat. no. A110-1), blood urea nitrogen (BUN; cat. no. C013-2) and creatinine (Cr; cat. no. C011-2) levels were analyzed using commercial kits (Nanjing KeyGen Biotech Co., Ltd., Nanjing, China). The levels of insulin (cat. no. CK-E30620), interleukin 2 (IL-2; cat. no. CK-E30648), interleukin 6 (IL-6; cat. no. CK-E30646), $\mathrm{NF}-\kappa \mathrm{B}$ (cat. no. CK-E91672R) and tumor necrosis factor- $\beta$ (TNF- $\beta$; cat. no. CK-E30527) were measured using the enzyme-linked immune sorbent assay method (Shanghai Yuan Ye Biotechnology Co. Ltd. Shanghai, China).

Statistical analysis. All data were expressed as the mean \pm standard error of the mean. Statistical significance was determined by a one-way analysis of variance followed by post-hoc multiple comparisons with Dunn's test, using SPSS 16.0 software (SPSS, Inc., Chicago, IL, USA). P $\leq 0.05$ was considered to indicate a statistically significant difference.

\section{Results}

Hypoglycemic effects of PTNE in diabetic rats. Changes in bodyweight, fasting blood glucose and serum insulin levels were measured to investigate the hypoglycemic effects of 
Table I. Bodyweight of rats in each group.

Body weight (g)

\begin{tabular}{lccccc}
\cline { 2 - 5 } Group & Day 0 & Week 8 & 3-day STZ injection & 2-week treatment & 4-week treatment \\
\hline Control & $165 \pm 5.21$ & $365.6 \pm 8.8$ & $367.1 \pm 4.1$ & $370.7 \pm 4.1$ & $376.7 \pm 9.0$ \\
DM & $168 \pm 6.8$ & $369.7 \pm 4.8$ & $314.6 \pm 18.9$ & $292.7 \pm 9.3^{\text {a }}$ & $297.1 \pm 10.3^{\mathrm{a}}$ \\
Met $(120 \mathrm{mg} / \mathrm{kg})$ & $170 \pm 7.9$ & $369.9 \pm 5.9$ & $319 \pm 10.1$ & $323.7 \pm 8.5^{\mathrm{b}}$ & $333 \pm 4.1^{\mathrm{c}}$ \\
PTNE $(\mathrm{g} / \mathrm{kg})$ & & & & & \\
0.04 & $167 \pm 4.3$ & $374.1 \pm 6.3$ & $293.8 \pm 16.3$ & $294.3 \pm 12.2$ & $294.6 \pm 8.1$ \\
0.20 & $169.8 \pm 5.7$ & $373.2 \pm 8.5$ & $296.3 \pm 13.5$ & $314.1 \pm 4.8$ & $323.4 \pm 7.5^{\mathrm{b}}$ \\
0.10 & $164 \pm 4.3$ & $369.1 \pm 9.8$ & $329.83 \pm 7.8$ & $321.4 \pm 12.9^{\mathrm{b}}$ & $331.4 \pm 10.7^{\mathrm{b}}$ \\
\hline
\end{tabular}

Data are expressed as the mean \pm standard error of the mean ( $\mathrm{n}=10$ /group). ${ }^{\mathrm{a}} \mathrm{P}<0.01$ vs. control. ${ }^{\mathrm{b}} \mathrm{P}<0.05$ and ${ }^{\mathrm{c}} \mathrm{P}<0.01$ vs. DM group. DM, diet/streptozotocin-induced diabetic rat model; Met, metformin; PTNE, Paecilomyces tenuipes N45 water extracts.
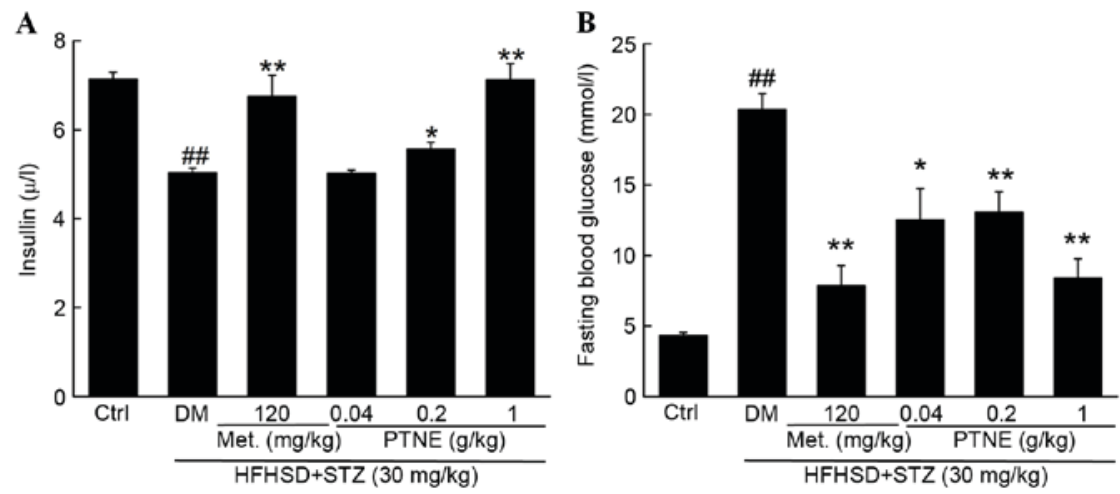

Figure 1. Following 4 weeks of Met and PTNE administration, the levels of (A) insulin and (B) fasting blood glucose in the serum of DM rats were detected. Data were expressed as the mean \pm standard error of the mean $(\mathrm{n}=10) .{ }^{\# \#} \mathrm{P}<0.01 \mathrm{vs}$. ctrl; ${ }^{*} \mathrm{P}<0.05$ and ${ }^{* *} \mathrm{P}<0.01$ vs. DM group. Ctrl, control; DM, diet/STZ-induced diabetic rat model; Met, metformin; PTNE, Paecilomyces tenuipes N45 water extracts; HFHSD, high fat and high sucrose diet; STZ, streptozotocin.

PTNE. Compared with the control group, decreased bodyweight and serum insulin levels and increased fasting blood glucose concentration were observed in diet/STZ-induced diabetic rats $(\mathrm{P}<0.01$; Table I). Normal body weight, insulin and fasting blood glucose levels were partially restored following 4 weeks of Met administration compared with the model group $(\mathrm{P}<0.01$; Fig. 1 and Table I). Similarly, PTNE treatment improved the body weight of diabetic rats, especially following 4 weeks of treatment $(\mathrm{P}<0.05$; Table I). Compared with the model group, $1 \mathrm{~g} / \mathrm{kg}$ PTNE treatment increased serum insulin levels by $\sim 41.5 \%$ ( $\mathrm{P}<0.01$; Fig. 1A). The fasting blood glucose concentration in the model group was increased by $11.1 \mathrm{mmol} / 1$ compared with the control group $(\mathrm{P}<0.01$; Fig. 1B) but $1 \mathrm{~g} / \mathrm{kg}$ PTNE treatment suppressed the fasting blood glucose levels by $58.7 \%$ compared with the model group $(\mathrm{P}<0.01$; Fig. 1B).

OGTT was used as a second diagnostic index to further confirm the hypoglycemic activities of PTNE. A significantly increased fasting blood glucose concentration was observed in the model group from 0.5 to $2.0 \mathrm{~h}$ compared with the control group ( $\mathrm{P}<0.001$; Fig. 2A) following oral glucose administration. Similar to Met, PTNE treatment partially prevented the increase of blood glucose levels at all chosen doses, but especially at $1.0 \mathrm{~g} / \mathrm{kg}$, compared with the model group $(\mathrm{P}<0.01$;
Fig. 2A). The calculated AUC values revealed an impaired glucose tolerance state in the model group compared with the control group $(\mathrm{P}<0.01$; Fig. 2B), but $0.04,0.2$ and $1.0 \mathrm{~g} / \mathrm{kg}$ PTNE and $120 \mathrm{mg} / \mathrm{kg}$ Met treatment all decreased the AUC compared with the model group $(\mathrm{P}<0.05$; Fig. 2B).

Hypolipidemic effects of PTNE in diabetic rats. Compared with the control group, TC (Fig. 3A), TG (Fig. 3B) and LDL-C levels (Fig. 3C) were increased in the model group $(\mathrm{P}<0.05)$, and HDL-C levels were decreased in the model group compared with the control group $(\mathrm{P}<0.05$, Fig. 3D). Treatment with $120 \mathrm{mg} / \mathrm{kg}$ Met significantly reduced TC and LDL-C serum levels compared with the model group $(\mathrm{P}<0.05)$, but failed to alter the levels of TG and HDL-C compared with the model group. However, PTNE treatment decreased TC, TG and LDL-C levels compared with the model group (Fig. 3A-C) and reversed the suppression of HDL-C levels in the serum of the model group $(\mathrm{P}<0.05$; Fig. 3D).

Anti-oxidative effects of PTNE in diabetic rats. Nutritional factors, including antioxidants, influence the management of diabetes mellitus and its complications (17). An imbalance between oxidative stress and antioxidative defense in diabetics accelerates diabetic complications (18). SOD and GSH-Px 

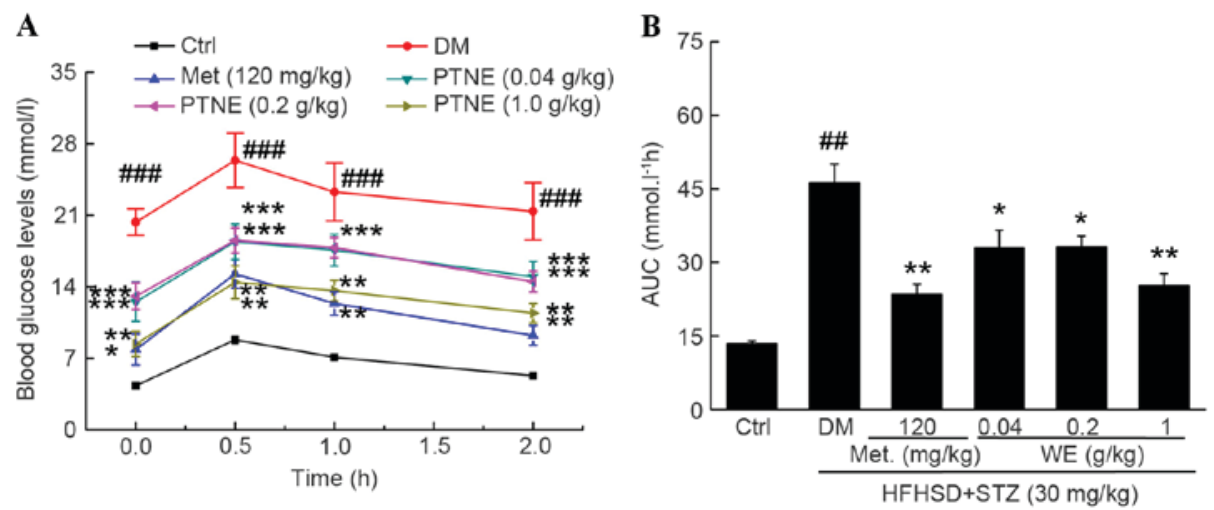

Figure 2. DM rats were treated with or without Met or PTNE for 4 weeks, and following oral administration of $2 \mathrm{~g} / \mathrm{kg}$ D-glucose, (A) the changes of plasma glucose from 0 to $2 \mathrm{~h}$ and (B) the area under the curve of glucose were analyzed. Data were expressed as the mean \pm standard error of the mean $(\mathrm{n}=10)$. ${ }^{\# t} \mathrm{P}<0.01$ and ${ }^{\# \# \#} \mathrm{P}<0.01$ vs. ctrl; ${ }^{*} \mathrm{P}<0.05,{ }^{* *} \mathrm{P}<0.01$ and ${ }^{* * *} \mathrm{P}<0.001$ vs. DM group. Ctrl, control; DM, diet/STZ-induced diabetic rat model; Met, metformin; PTNE, Paecilomyces tenuipes N45 water extracts; HFHSD, high fat and high sucrose diet; STZ, streptozotocin.
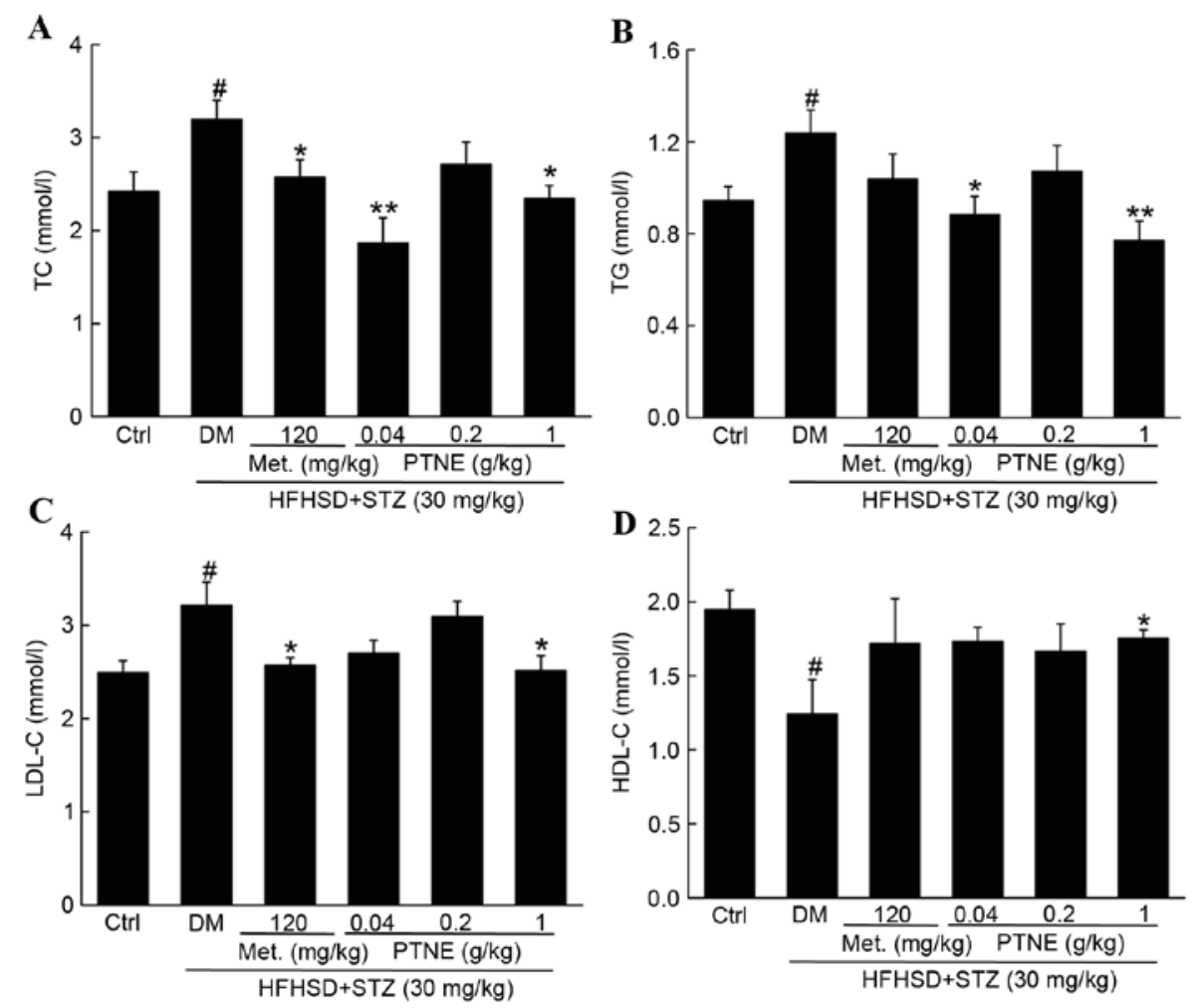

Figure 3. Beneficial effects of Met and PTNE treatment on (A) TC, (B) TG, (C) LDL-C and (D) HDL-C in DM rats. Data are expressed as the mean \pm standard error of the mean $(\mathrm{n}=10)$. ${ }^{*} \mathrm{P}<0.05$ vs.ctrl; ${ }^{*} \mathrm{P}<0.05$ and ${ }^{* * *} \mathrm{P}<0.01$ vs. DM group. Met, metformin; PTNE, Paecilomyces tenuipes $\mathrm{N} 45$ water extracts; TC, total cholesterol; Ctrl, control; DM, diet/STZ-induced diabetic rat model; Met, metformin; PTNE, Paecilomyces tenuipes N45 water extracts; HFHSD, high fat and high sucrose diet; STZ, streptozotocin; TG, triglycerides; LDL-C, low-density lipoprotein cholesterol; HDL-C, low-density lipoprotein cholesterol.

activity levels were decreased in the model group compared with the control group $(\mathrm{P}<0.05$; Table II), and overproduction of MDA serum levels were increased in the model group compared with the control group ( $\mathrm{P}<0.05$; Table II). Similar to Met treatment, PTNE partially restored SOD, GSH-Px and MDA serum levels, especially at the dose of $1 \mathrm{~g} / \mathrm{kg}(\mathrm{P}<0.05$; Table II).

Anti-nephropathic effects of PTNE in diabetic rats. BUN and Crare recognized as sensitive indexes for kidney injury (19) and BUN and $\mathrm{Cr}$ levels were significantly increased in the model group compared with the control group $(\mathrm{P}<0.05$; Fig. 4A and B). Treatment with Met and PTNE for 4 weeks significantly attenuated this increase in $\mathrm{BUN}(\mathrm{P}<0.05$; Fig. 4A) and $\mathrm{Cr}(\mathrm{P}<0.05$; Fig. 4B). Severe inflammation occurred in the model group and was characterized by the release of multiple inflammatory factors, including increased serum levels of IL-2 ( $\mathrm{P}<0.05$; Fig. 4C), IL-6 ( $<<0.05$; Fig. 4D) and TNF- $\beta(\mathrm{P}<0.05$; Fig. 4E) compared with the control group. Met and PTNE treatment significantly suppressed these indexes, with IL-2 (Fig. 4C) IL-6 (Fig. 4D) and TNF- $\beta$ (Fig. 4E) serum levels significantly decreased compared 
Table II. Met and PTNE treatment increased SOD and GSH-Px activity, and reduced MDA serum levels of DM rats.

\begin{tabular}{lccc}
\hline Group & SOD $(\mu / \mathrm{ml})$ & GSH-Px $(\mu \mathrm{mol} / \mathrm{l})$ & MDA $(\mathrm{nmol} / \mathrm{ml})$ \\
\hline Control & $368.4 \pm 30.4$ & $246.9 \pm 17.7$ & $6.2 \pm 1.5$ \\
DM & $196.3 \pm 18.5^{\mathrm{b}}$ & $176.1 \pm 22.3^{\mathrm{a}}$ & $23.5 \pm 4.9^{\mathrm{b}}$ \\
Met $(120 \mathrm{mg} / \mathrm{kg})$ & $316.5 \pm 30.9^{\mathrm{d}}$ & $254.5 \pm 27.1^{\mathrm{c}}$ & $9.7^{\mathrm{b}}$ \\
PTNE $(\mathrm{g} / \mathrm{kg})$ & & & $2.1^{\mathrm{c}}$ \\
0.04 & $256.1 \pm 24.6$ & $241.6 \pm 15.5^{\mathrm{c}}$ & $25.3 \pm 3.7$ \\
0.20 & $280.9 \pm 13.4^{\mathrm{c}}$ & $202.6 \pm 17.2$ & $22.2 \pm 4.6$ \\
0.10 & $264.5 \pm 17.5^{\mathrm{c}}$ & $218.1 \pm 10.8$ & $11.7 \pm 1.7^{\mathrm{c}}$ \\
\hline
\end{tabular}

Data were expressed as the mean \pm standard error of the mean ( $\mathrm{n}=10 /$ group). ${ }^{\mathrm{a}} \mathrm{P}<0.05$ and ${ }^{\mathrm{b}} \mathrm{P}<0.01$ vs. control. ${ }^{\mathrm{c}} \mathrm{P}<0.05$ and ${ }^{\mathrm{d}} \mathrm{P}<0.01$ vs. $\mathrm{DM}$ group. Met, metformin; PTNE, Paecilomyces tenuipes N45 water extracts; SOD, superoxide dismutase; GSH-Px, glutathione peroxidase; MDA, methane dicarboxylic aldehyde; DM, diet/streptozotocin-induced diabetic rat model.
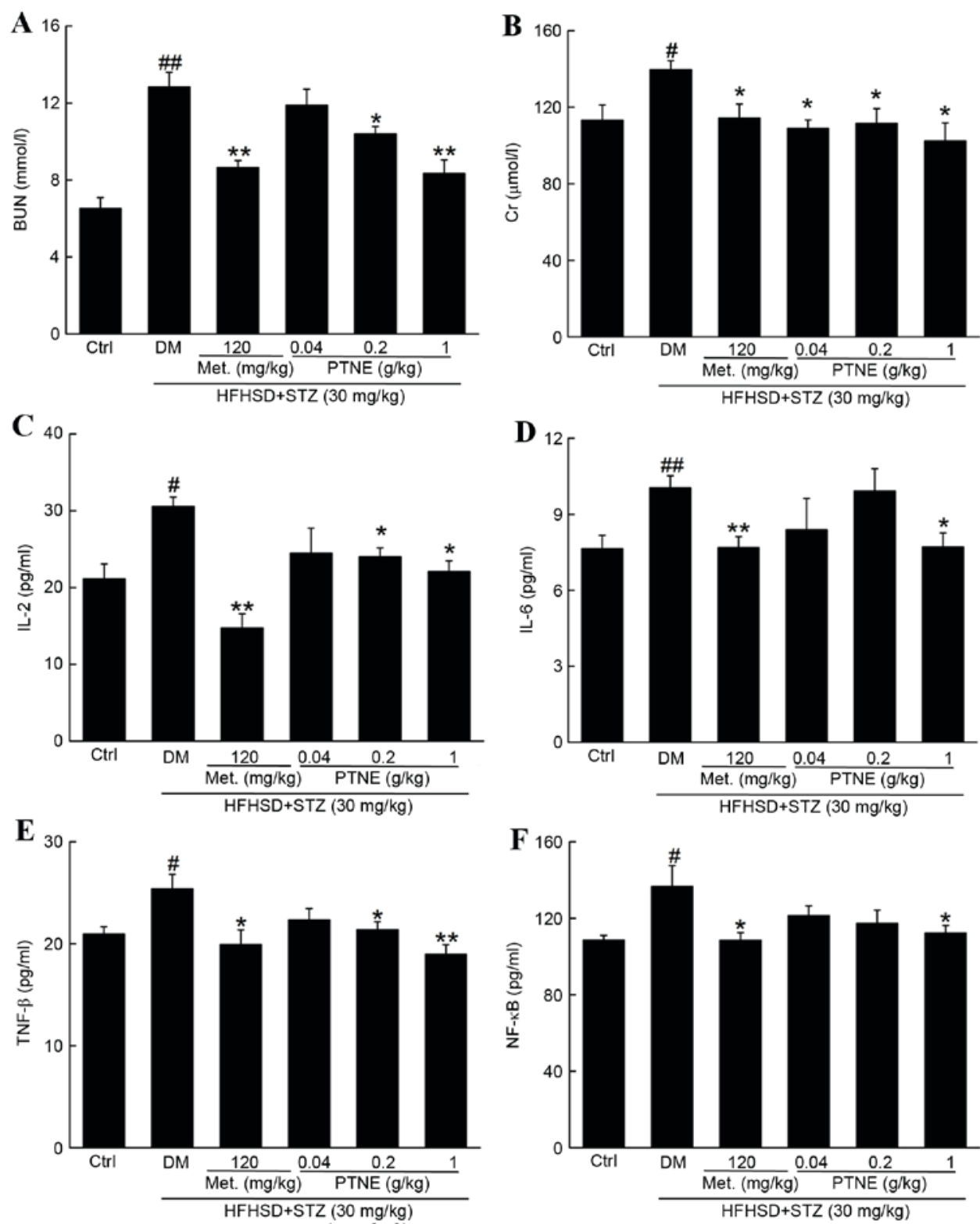

Figure 4. Anti-nephropathic effects of PTNE treatment in DM rats. The serum levels of (A) BUN, (B) Cr, (C) IL-2, (D) IL-6, (E) TNF- $\beta$ and (F) NF- $\mathrm{kB}$ were determined via ELISA. Data are expressed as the mean \pm standard error of the mean $(n=10) .{ }^{*} \mathrm{P}<0.05$ and ${ }^{\# t} \mathrm{P}<0.01$ vs. ctrl; ${ }^{*} \mathrm{P}<0.05$ and ${ }^{* * *} \mathrm{P}<0.01$ vs. DM group. PTNE, Paecilomyces tenuipes N45 water extracts; DM, diet/STZ-induced diabetic rat model; BUN, blood urea nitrogen; Ctrl, control; DM, diet/STZ-induced diabetic rat model; Met, metformin; PTNE, Paecilomyces tenuipes N45 water extracts; HFHSD, high fat and high sucrose diet; STZ, streptozotocin; Cr,

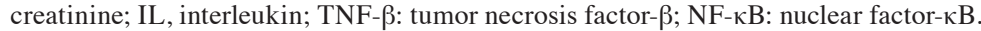


with the model group $(\mathrm{P}<0.05)$. Notably, $120 \mathrm{mg} / \mathrm{kg}$ Met and $1.0 \mathrm{~g} / \mathrm{kg}$ PTNE treatment decreased NF- $\mathrm{kB}$ serum levels by 20.5 and $17.8 \%$, respectively, compared with the model group (P<0.05; Fig. 4F).

\section{Discussion}

The rat model of diabetes, induced by a high-fat diet and a low dose of STZ, displays similar metabolic features to those of human type 2 diabetes mellitus (20), which is a complex and heterogeneous disorder associated with a progressive decline in insulin action.

Previous research has focused on the use of traditional folk medicines produced from natural plants, which has resulted in significant developments in functional food, nutraceuticals and pharmaceuticals $(21,22)$. In contrast to the majority of treatments used to manage diabetes, as a crude fungus, $P$. tenuipes contains a complex mixture of bioactive components, which have multiple molecular targets. This multipronged targeting approach may reduce hyperglycemia, inflammation and oxidative stress in a more natural way, and may result in fewer adverse side effects. Based on the findings of the present study and its toxicological profile (23), P. tenuipes N45 has been demonstrated to be both safe and effective.

As an agent for inducing diabetes, STZ causes $\beta$-cell injury in the pancreas (24). The pancreas is responsible for regulating serum glucose concentration. The anti-hyperglycemic activity of natural products usually occurs due to the restoration of pancreatic function via the enhancement of insulin secretion (7). In the present study, PTNE increased serum insulin levels and demonstrated positive activity on OGTT, which is an important index for evaluating islet function (25). The beneficial effect on pancreatic function may be an important contributor to the hypoglycemic effect of PTNE.

Altered lipid metabolism, including changes in TG, TC, HDL-C and LDL-C levels, are observed in type 2 diabetes mellitus (26). This is a risk factor for coronary heart disease (27). PTNE may be beneficial in preventing coronary heart disease and atherosclerosis via the regulation of lipid metabolism. During development of diabetes mellitus, insulin resistance is responsible for the release of adipocytokines and relaxation of the afferent arteriole. The risk of the development of atherosclerosis in diabetes mellitus can be reduced by elevating HDL-C levels (28). On the other hand, diabetesassociated dyslipidemia results in lipid accumulation in the kidney, which leads to insulin resistance, inflammation and oxidative stress (29). The antilipemic effects of PTNE may be involved in its anti-diabetic and anti-nephropathic activities.

Diabetic nephropathy represents a risk factor for mortality, including cardiovascular mortality (30). Suppressed BUN and creatinine levels, which are important markers of kidney damage, were observed in PTNE-treated diabetic rats. It has previously been reported that chronic inflammation during the development of diabetes contributes to nephropathy (31). Interleukins, especially IL-2 and IL-6, are involved in glomerular damage (32). Furthermore, high glucose levels initiate an inflammatory response characterized by the activation of the pro-inflammatory NF- $\mathrm{BB}$ pathway, which promotes the expression and activity of its downstream inflammatory mediators (33). Based on the results of the present study, the renal protective activity of PTNE against diabetic nephropathy has been confirmed.

Hyperglycemia leads to accumulation of ROS, which in turn mediate various metabolic defects associated with the diabetic state (29). An imbalance between oxidative stress and antioxidative defense in diabetes results in cell and tissue damages and accelerate diabetic complications (18). SOD and GSH-Px are regarded as the primary defense systems against ROS generation (34). MDA is a major product of lipid peroxidation, and low levels of MDA suggest reduced lipid peroxidation and weaker oxidant stress (32). Anti-oxidant agents are emerging as potential agents for preventing pancreatic $\beta$ cell destruction (28). Therefore, the antioxidative effects of PTNE may be another important contributor to its modulation of blood glucose and lipid metabolism and inflammation.

In summary, in diet/STZ-induced diabetic rats, the hypolipidemic, hypoglycemic and anti-diabetic nephritic effects of PTNE have been confirmed. Previous data suggest that all these effects may be associated with to the modulation of oxidative damages by PTNE. The findings of the present study support the use of PTNE as a pharmaceutical agent for the management of diabetes and diabetic complications.

\section{Acknowledgements}

The present study was supported by the Science and Technology Key Project in Jilin Province of China (grant nos. 20140311072YY, 20150203002NY, 20160520036JH and 20160204029YY).

\section{References}

1. Reagan LP: Diabetes as a chronic metabolic stressor: Causes, consequences and clinical complications. Exp Neurol 233: 68-78, 2012.

2. Kerner W, Bruckel J; German Diabetes Association: Definition, classification and diagnosis of diabetes mellitus. Exp Clin Endocrinol Diabetes 122: 384-386, 2014.

3. Winkler G, Hidvegi T, Vandorfi G, Balogh S and Jermendy G: Risk-stratified screening for type 2 diabetes in adult subjects: Results from hungary. Diabetologia 54: S119-S119, 2011.

4. Tuttle KR: Linking metabolism and immunology: Diabetic nephropathy is an inflammatory. J Am Soc Nephrol 16: $1537-1538,2005$

5. Fornoni A, Ijaz A, Tejada T and Lenz O: Role of inflammation in diabetic nephropathy. Curr Diabetes Rev 4: 10-17, 2008.

6. Kania DS, Gonzalvo JD and Weber ZA: Saxagliptin: A clinical review in the treatment of type 2 diabetes mellitus. Clin Ther 33: 1005-1022, 2011.

7. Malviya N, Jain S and Malviya S: Antidiabetic potential of medicinal plants. Acta Pol Pharm 67: 113-118, 2010.

8. Zhang G, Huang Y, Bian Y, Wong JH, Ng TB and Wang H: Hypoglycemic activity of the fungi Cordyceps militaris, Cordyceps sinensis, Tricholoma mongolicum, and Omphalia lapidescens in streptozotocin-induced diabetic rats. Appl Microbiol Biotechnol 72: 1152-1156, 2006.

9. Fukatsu T, Sato H and Kuriyama H: Isolation, inoculation to insect host, and molecular phylogeny of an entomogenous fungus Paecilomyces tenuipes. J Invertebr Pathol 70: 203-208, 1997.

10. Yin YY, Ming L, Zheng LF, Kan HW, Li CR and Li WP: Bioactive compounds from Paecilomyces tenuipes regulating the function of the hypothalamo-hypophyseal system axis in chronic unpredictable stress rats. Chin Med J (Engl) 120: 1088-1092, 2007.

11. Lee DH, Park T and Kim HW: Induction of apoptosis by disturbing mitochondrial-membrane potential and cleaving PARP in Jurkat T cells through treatment with acetoxyscirpenol mycotoxins. Biol Pharm Bull 29: 648-654, 2006. 
12. Lee SM, Park NS, Jin BR, Kang HS, Jung JH and Park E: Effects of Paecilomyces tenuipes cultivated in egg yolk on lipid metabolism in rats on a high fat-cholesterol diet. J Med Food 9: 214-222, 2006.

13. Du L, Liu C, Teng M, Meng Q, Lu J, Zhou Y, Liu Y, Cheng Y, Wang D and Teng L: Antidiabetic activities of Paecilomyces tenuipes N45 extract in alloxan-induced diabetic mice. Mol Med Rep 13: 1701-1708, 2016.

14. Balamurugan K, Nishanthini A and Mohan VR: Antidiabetic and antihyperlipidaemic activity of ethanol extract of Melastoma malabathricum Linn. leaf in alloxan induced diabetic rats. Asian Pac J Trop Biomed 4 (Suppl 1): S442-S448, 2014.

15. Nakahara Y, Ozaki K, Sano T, Kodama Y and Matsuura T: Assessment of alloxan-induced diabetic rats as a periodontal disease model using a selective cyclooxygenase (COX)-2 inhibitor. J Toxicol Pathol 27: 123-129, 2014.

16. Bajaj M, Suraamornkul S, Pratipanawatr T, Hardies LJ, Pratipanawatr W, Glass L, Cersosimo E, Miyazaki Y and DeFronzo RA: Pioglitazone reduces hepatic fat content and augments splanchnic glucose uptake in patients with type 2 diabetes. Diabetes 52: 1364-1370, 2003.

17. Park JH, Park NS, Lee SM and Park E: Effect of dongchunghacho rice on blood glucose level, lipid profile, and antioxidant metabolism in streptozotocin-induced diabetic rats. Food Sci Biotechnol 20: 933, 2011.

18. Luo Q, Cai YZ, Yan J, Sun M and Corke H: Hypoglycemic and hypolipidemic effects and antioxidant activity of fruit extracts from Lycium barbarum. Life Sci 76: 137-149, 2004.

19. Zhu S, Wang J, Zhang Y, Li V, Kong J, He J and Li XM: Unpredictable chronic mild stress induces anxiety and depression-like behaviors and inactivates AMP-activated protein kinase in mice. Brain Res 1576: 81-90, 2014

20. Srinivasan K, Viswanad B, Asrat L, Kaul CL and Ramarao P: Combination of high-fat diet-fed and low-dose streptozotocin-treated rat: A model for type 2 diabetes and pharmacological screening. Pharmacol Res 52: 313-320, 2005.

21. Xu W, Zhou Q, Yin JJ, Yao Y and Zhang JL: Anti-diabetic effects of polysaccharides from Talinum triangulare in streptozotocin (STZ)-induced type 2 diabetic male mice. Int J Biol Macromol 72: 575-579, 2015

22. He J and Li YL: Ginsenoside Rg1 downregulates the shear stress induced MCP-1 expression by inhibiting MAPK signaling pathway. Am J Chin Med 43: 305-317, 2015.

23. Du L, Liu Y, Liu C, Meng Q, Song J, Wang D, Lu J, Teng L, Zhou Y and Teng L: Acute and subchronic toxicity studies on safety assessment of Paecilomyces tenuipes N45 extracts. Comb Chem High Throughput Screen 18: 809-818, 2015.
24. Wei L, Lu Y,He S, Jin X, Zeng L, Zhang S, Chen Y, Tian B, Mai G, Yang G, et al: Induction of diabetes with signs of autoimmunity in primates by the injection of multiple-low-dose streptozotocin. Biochem Biophys Res Commun 412: 373-378, 2011.

25. Buchanan TA, Xiang AH, Peters RK, Kjos SL, Marroquin A, Goico J, Ochoa C, Tan S, Berkowitz K, Hodis HN and Azen SP: Preservation of pancreatic beta-cell function and prevention of type 2 diabetes by pharmacological treatment of insulin resistance in high-risk hispanic women. Diabetes 51: 2796-2803, 2002.

26. Arvind K, Pradeepa R, Deepa R and Mohan V: Diabetes \& coronary artery disease. Indian J Med Res 116: 163-176, 2002.

27. Sakatani T, Shirayama T, Suzaki Y, Yamamoto T, Mani H, Kawasaki T, Sugihara $\mathrm{H}$ and Matsubara $\mathrm{H}$ : The association between cholesterol and mortality in heart failure. Comparison between patients with and without coronary artery disease. Int Heart J 46: 619-629, 2005.

28. Vasan S, Foiles P and Founds H: Therapeutic potential of breakers of advanced glycation end product-protein crosslinks. Arch Biochem Biophys 419: 89-96, 2003.

29. Rolo AP and Palmeira CM: Diabetes and mitochondrial function: Role of hyperglycemia and oxidative stress. Toxicol Appl Pharm 212: 167-178, 2006.

30. Targher G, Zoppini G, Chonchol M, Negri C, Stoico V, Perrone F, Muggeo M and Bonora E: Glomerular filtration rate, albuminuria and risk of cardiovascular and all-cause mortality in type 2 diabetic individuals. Nutr Metab Cardiovas Dis 21: 294-301, 2011.

31. Chow FY, Nikolic-Paterson DJ, Atkins RC and Tesch GH: Macrophages in streptozotocin-induced diabetic nephropathy: Potential role in renal fibrosis. Nephrol Dial Transplant 19: 2987-2996, 2004.

32. Padmavathi R, Senthilnathan P, Chodon D and Sakthisekaran D: Therapeutic effect of paclitaxel and propolis on lipid peroxidation and antioxidant system in 7,12 dimethyl benz(a) anthracene-induced breast cancer in female Sprague Dawley rats. Life Sci 78: 2820-2825, 2006

33. Pan Y, Wang Y, Cai L, Cai Y, Hu J, Yu C, Li J, Feng Z, Yang S, Li X and Liang G: Inhibition of high glucose-induced inflammatory response and macrophage infiltration by a novel curcumin derivative prevents renal injury in diabetic rats. Br J Pharmacol 166: 1169-1182, 2012.

34. Kim JO, Kim KS, Lee GD and Kwon JH: Antihyperglycemic and antioxidative effects of new herbal formula in streptozotocininduced diabetic rats. J Med Food 12: 728-735, 2009. 